\title{
Quality of Life in Cardiovascular Surgery: Elaboration and Initial Internal Validation of a Quality of Life Questionnaire
}

Marina Macedo Kuenzer Bond', MD; Jenny Lourdes Rivas de Oliveira', MD; Luiz Carlos Bento de Souza', MD, PhD; Pedro Silvio Farsky', MD, PhD; Vivian Lerner Amato', MD, PhD; Dorival Julio Della Togna', MD, PhD; Samira Kaissar Ghorayeb', MD; Magaly Arrais dos Santos', MD, PhD

DOI: $10.21470 / 1678-9741-2018-0108$

\begin{abstract}
Objective: Elaboration and internal validation of the Quality of Life in Cardiovascular Surgery (QLCS) questionnaire adapted to the reality of Brazilian cardiovascular surgery.

Methods: Cross-sectional pilot study of a prospective cohort included in the Documentation and Surgical Registry Center (CEDREC) for internal validation of the QLCS questionnaire. Four hundred forty-five patients submitted to cardiovascular surgery and who answered a QLCS questionnaire 30 days after hospital discharge were included. It was applied via telephone. To verify the questions' internal consistency, Cronbach's alpha was used. The total QLCS score was calculated as the sum of 5 questions, ranging from 5 to 25 points. Mann-U-Whitney test was used to relate the symptoms with the quality of life (QoL). Level of significance was $\mathbf{5 \%}$.
\end{abstract}

Results: After 30 days of surgery, about $95 \%$ of the patients had already returned to normal routine and $19 \%$ of them were already performing physical activity. In the evaluation of the QLCS's internal consistency, a Cronbach's alpha of 0.74 was found, suggesting that this was probably an adequate questionnaire to evaluate QoL in this population. In the comparison between the presence and absence of symptoms and the median of QoL, the presence of pain at the incision $(P=0.002)$, chest pain $(P<0.001)$, shortness of breath $(P<0.001)$, and return to physical activity $(P<0.001)$ were statistically significant.

Conclusion: The process of elaboration and validation of questionnaires includes a series of steps. The QLCS questionnaire is probably an adequate tool for the evaluation of QoL in the postoperative patient of cardiovascular surgery, in this first stage of internal validation.

Keywords: Quality of Life. Cardiovascular Surgical Procedures. Coronary Artery Bypass.

\begin{tabular}{ll} 
Abbreviations, acronyms \& symbols \\
\hline $\begin{array}{ll}\text { CABG } & =\text { Coronary artery bypass grafting } \\
\text { CEDREC } & =\text { Documentation and Surgical Registry Center } \\
\text { KCCQ } & =\text { Kansas City Cardiomyopathy Questionnaire } \\
\text { LQ } & =\text { Lower quartile } \\
\text { MOS SF-36 or SF-36 } & =\text { Medical Outcomes Study 36-Item Short-Form Health Survey } \\
\text { QLCS } & =\text { Quality of Life in Cardiovascular Surgery } \\
\text { QoL } & =\text { Quality of life } \\
\text { SF-12 } & =\text { Short version of MOS SF-36 or SF-36 } \\
\text { UQ } & =\text { Upper quartile } \\
\text { WHOQOL-100 } & =\text { The World Health Organization Quality of Life Assessment } \\
\text { WHOQOL-BREF } & =\text { Summary version of WHOQOL-100 } \\
\text { WHOQOL-group } & =\text { The World Health Organization Quality of Life Group }\end{array}$
\end{tabular}

'Instituto Dante Pazzanese de Cardiologia, São Paulo, Brazil.

This study was carried out at the Instituto Dante Pazzanese de Cardiologia, São Paulo, Brazil.

No financial support.

No conflict of interest.
Correspondence Address:

Marina Macedo Kuenzer Bond

Instituto Dante Pazzanese de Cardiologia

Av. Dr. Dante Pazzanese, 500 - Vila Mariana - São Paulo, SP, Brazil

Zip code: 04012-909

E-mail:mmkbond@gmail.com 


\section{INTRODUCTION}

Traditionally defined by philosophers and poets, quality of life (QoL) and its measurement are becoming a fundamental topic for the practice of Medicine ${ }^{[1]}$.

According to The World Health Organization Quality of Life Group (WHOQOL-group), QoL is defined as an individual's perception of his/her position in life in the context of the culture and value systems in which he/she lives and in relation to his/ her goals, expectations, standards, and concerns. It is a broad ranging concept affected in a complex way by the person's physical health, psychological state, personal beliefs, social relationships, and his/her relationship to salient features of his/ her environment ${ }^{[2]}$.

The impact of any event or health status on a person's QoL depends to a large extent on his/her personal projects, desires, and history ${ }^{[1]}$. Although the most important indicator of health status is the clinical outcome, QoL and patient satisfaction are key indicators for the adequate understanding and treatment of pathologies ${ }^{[3]}$. The QoL related to cardiovascular disease and the impact of the treatment on each patient's life have been researched, contributing to the clinical decision-making process, as well as improving patient care, aiming at a more integral approach to health (physical, psychological, and social) ${ }^{[4]}$.

To evaluate QoL, you can choose to use generic questionnaires, which do not specify the disease itself, such as the Medical Outcomes Study 36-Item Short-Form Health Survey (MOS SF-36 or SF-36) ${ }^{[5]}$, and its short version 12 (SF-12) ${ }^{[6]}$, or The World Health Organization Quality of Life Assessment $(\mathrm{WHOQOL-100)})^{[7]}$, and its summary version (WHOQOL-BREF $)^{[8]}$, or specific questionnaires, developed for a particular pathology, such as the Kansas City Cardiomyopathy Questionnaire (KCCQ) for heart failure ${ }^{[9]}$.

Improving QoL is one of the main objectives for cardiovascular surgery, so a review on this subject was published in 2011, which included 29 articles published between January 2004 and December 2010, but only nine studies presented data on pre and postoperative QoL, and that was a limitation ${ }^{[10]}$. Another meta-analysis published in $2013^{[11]}$ selected 15 articles on QoL in the postoperative period of cardiovascular surgery, and most of the analyzed studies showed a significant improvement in QoL in the evaluation instruments; the SF-36 was the most used questionnaire in $43 \%$ of the studies.
Therefore, QoL measurement of the postoperative period of cardiovascular surgery is crucial to evaluate the patients'treatment, and the creation and validation of a current questionnaire which is more adequate for these patients' reality is of fundamental importance. The objectives of this study were to create, based on an adaptation of the main existing QoL questionnaires, and to validate a current and adequate questionnaire, easy to apply, which can be performed via telephone, for the reality of the postoperative period of cardiovascular surgery in Brazil.

\section{METHODS}

This is a pilot study for initial validation of the Quality of Life in Cardiovascular Surgery (QLCS) questionnaire, using as sample the population included in the Documentation and Surgical Registry Center (CEDREC) of a hospital specialized in Cardiology. CEDREC is a computerized database specifically for patients undergoing cardiovascular surgery in our hospital. Patients who were operated on and accepted to participate in the database were included and monitored prospectively.

This cross-sectional study taken from a prospective cohort included patients who underwent cardiovascular surgery, from July 2016 until October 2017, who accepted to participate in CEDREC, and who properly answered a QoL questionnaire (the QLCS) after thirty days of hospital discharge. Only patients under 18 years old were excluded. All the other patients were included in the sample.

The questionnaire was created by a group of medical specialists, based on the main existing QoL questionnaires (SF-36, SF-12, WHOQOL-100, WHOQOL-BREF), and adapted to the current reality of Brazilian medicine. It is a simple and fast questionnaire, easy to perform, and feasible to be applied via telephone. The questionnaire used by CEDREC had 21 questions (Table 1), being five questions on QoL, which correspond to the QLCS questionnaire (Table 2), and the others were about medication, symptoms, procedures, and internationalization in that period. These five QLCS questions were created and considered adequate and clear enough by all medical experts to compose a questionnaire whose main objective is to easily and quickly assess patients' QoL in the postoperative period of cardiovascular surgery. The total QoL score was calculated as the sum of the five QoL questions, ranging from 5 to 25 points. The higher the value found, the better the QoL.

Table 1. Complete questionnaire used by the Documentation and Surgical Registry Center (CEDREC) (English version).

This questionnaire asks for your opinion about your health and how you feel and about your ability to perform your daily activities in the last period (30 days, 6 months, 12 months, and annually).

1) Has the patient taken any tests in the last period?

\begin{tabular}{l|l|l|l|l|l}
\hline Yes & No & Does not know & Not applicable & & \\
\hline 2) Has the patient had any intervention in the last period? & Does not know & Not applicable & & \\
\hline Yes & No & Dot & & \\
\hline 3) Has the patient had any surgical procedure in the last period? & Does not know & Not applicable & & \\
\hline Yes & No & \\
\hline
\end{tabular}


4) Is the patient taking any medication?

\begin{tabular}{l|l|l|l|l|l}
\hline Yes & No & Does not know & Not applicable & \multicolumn{1}{l}{} & \\
\hline 5) Does the patient feel pain in the surgical incision? & Does not know & Not applicable & & \\
\hline Ys & No & \multicolumn{1}{l}{} \\
\hline 6) Has the patient had any infection? & No & Does not know & Not applicable & & \\
\hline Yes & No & Does not know & Not applicable & & \\
\hline 7) Does the patient feel chest pain? & Yes &
\end{tabular}

8) Does the patient experience shortness of breath?

\begin{tabular}{l|l|l|l|l|l}
\hline Yes & No & Does not know & Not applicable & & \\
\hline 9) Did the patient have other symptoms? & Does not know & Not applicable & & \\
\hline Yes & No & D
\end{tabular}

10) Does the patient do physical activity?

\begin{tabular}{l|l|l|l|l|l}
\hline Yes & No & Does not know & Not applicable & & \\
\hline 11) Did the patient return to normal work/school/activities? & & \\
\hline Yes & No & Does not know & Not applicable & & \\
\hline
\end{tabular}

12) How is the patient's performance in daily activities/work/school?

\begin{tabular}{l|l|l|l|l|l}
\hline Too bad & Bad & Good & Very good & Great & Not applicable \\
\hline 13) How is the patient's health after surgery? & Good & Very good & Great & Not applicable \\
\hline Too bad & Bad
\end{tabular}

14) How is the patient's physical capacity after surgery?

\begin{tabular}{l|l|l|l|l|l}
\hline Too bad & Bad & Good & Very good & Great & Not applicable \\
\hline 15) From the emotional point of view, how is the patient feeling? & Good & Very good & Great & Not applicable \\
\hline Too bad & Bad &
\end{tabular}

16) In the relationship with his/her family members, how is the patient feeling?

\begin{tabular}{l|l|l|l|l|l}
\hline Too bad & Bad & Good & Very good & Great & Not applicable \\
\hline 17) Did the patient have chest trauma? & Not applicable & & & & \\
\hline Yes & No & & & \\
\hline
\end{tabular}

18) Did the patient have fainting episodes?

\begin{tabular}{l|l|l|l|l|l}
\hline Yes & No & Not applicable & & \\
\hline 19) Did the patient feel palpitations? & No & Not applicable & & & \\
\hline Yes & No & & \\
\hline 20) Did the patient have fall and suffer cranial trauma? & Not applicable & & & \\
\hline Yes & no & & & \\
\hline 21) Did the patient gain or lose weight? & Not applicable & & & \\
\hline Yes & NO & & \\
\hline
\end{tabular}

After the creation of any questionnaire, it must go through an initial validation step with a pilot sample. In this pilot test, the questionnaire's final version is administered to a large representative sample of respondents for whom the questionnaire is intended. At this point, it is important to evaluate the questionnaire's reliability. This is accomplished by assessing the internal consistency and testing - retesting reliability. Internal consistency reflects the extent to which the questionnaire items are intercorrelated or whether they are consistent in measurement of the same construct ${ }^{[12]}$. Internal consistency is commonly estimated using the alpha coefficient, also known as Cronbach's alpha ${ }^{[13]}$.

For statistical analysis, Cronbach's alpha was used to verify the questionnaire's internal consistency and to validate the 
Table 2. Quality of Life in Cardiovascular Surgery (QLCS) questionnaire (English version)

This questionnaire asks for your opinion about your health and how you feel and about your ability to perform your daily activities in the last 30 days, 6 months, 12 months, and annually.

\begin{tabular}{|c|c|c|c|c|}
\hline Answer the & ns as foll & & & \\
\hline 1 - Too Bad & 2 - Bad & 3 - Good & 4 - Very Good & 5-Great \\
\hline
\end{tabular}

1) How is the patient's performance in daily activities/work/school?

2) How is the patient's health after surgery?

3) How is the patient's physical capacity after surgery?

4) From the emotional point of view, how is the patient feeling?

5) In the relationship with his/her family members, how is the patient feeling?

Total / 25 points

questionnaire. Assuming a standard deviation of approximately 6 units and a detectable difference of 3 points, with 5\% of alpha error and $95 \%$ of power, it would be required a total of 63 cases. The quantitative variables were described by mean and standard deviation in the presence of normal distribution, or median and interquartile range in the presence of asymmetric distribution. The qualitative variables will be presented by absolute frequencies (number of patients) and relative frequencies (percentages). The Mann-U-Whitney test was used to relate the symptoms (qualitative variable) with the QoL (quantitative variable). Level of significance was set at $5 \%$.

\section{RESULTS}

Four hundred forty-five patients submitted to cardiovascular surgery, from July 2016 to October 2017, and who had a QoL questionnaire answered 30 days after hospital discharge were included. The mean age of the sample was 60.20 years $( \pm 13.29)$, of which $58.43 \%$ were males and $41.57 \%$ were females.

The initial characteristics of the sample are summarized in Table 3, but we can highlight that $29.6 \%$ of them were diabetic, $60 \%$ were hypertensive, $34.6 \%$ were dyslipidemic, and $28.5 \%$ had a history of smoking.

About the surgeries performed, the largest part consisted of valve surgeries, with a total of 198 (44\%) cases, followed by coronary artery bypass grafting (CABG) with 164 (37\%) cases, aortic surgeries with 39 (8.7\%) cases, and congenital anomaly correction surgery with 19 (4.2\%) cases. The remainder comprised of $6(1.3 \%)$ cases of heart transplant surgeries, $7(1.5 \%)$ cases of pericardiectomies, $4(0.9 \%)$ cases of myectomies, $3(0.6 \%)$ cases of tumor removals, and 9 (2\%) cases of reshaping and cleaning. It is noteworthy that in some cases more than one type was performed at the same surgery time (Figure 1).

After 30 days of surgery, $35 \%$ of the patients had incision pain, $17 \%$ wound infection, $23 \%$ chest pain, $22 \%$ lack of air, $1 \%$ fainting, 5\% palpitation, and $43 \%$ other symptoms. However, 95\% of the patients had already returned to normal routine and $19 \%$ were already performing physical activity.

In the evaluation of the QLCS's internal consistency, a Cronbach's alpha of 0.74 was found, proving this to be a questionnaire with adequate internal consistency. This means that, in the evaluation of the degree of variation of these questions, it was demonstrated that the items are appropriately related to each other.

When comparing between patients with and without incision pain after 30 days of surgery, we observed that patients with this symptom presented a median of 19.0 in the QLCS questionnaire, with lower quartile (LQ) of 16.0 and upper quartile (UQ) of 22.0, while those without incision pain presented a median of $20.0(\mathrm{LQ}=18.0 ; \mathrm{UQ}=22$ ). When using the Mann- $\mathrm{U}$ Whitney test, this observed reduction was significant $(P=0.002)$. The same was also observed for patients with chest pain, with a median of $18.50(\mathrm{LQ}=16.0 ; \cup Q=21.0)$, when comparing with those without chest pain, with a median of 20.0 ( $L Q=18.0$; $\cup Q=22.0)$; significant difference $(P<0.001)$. The presence of

Table 3. Sample's characteristics.

\begin{tabular}{l|c|c}
\hline & N & $\%$ \\
\hline Female & 185 & 41.57 \\
\hline Male & 260 & 58.43 \\
\hline Diabetes & 132 & 29.66 \\
\hline Arterial hypertension & 267 & 60.00 \\
\hline Dyslipidemia & 154 & 34.60 \\
\hline Smoker & 127 & 28.53 \\
\hline Chronic obstructive pulmonary disease & 10 & 2.24 \\
\hline Rheumatic fever & 30 & 6.74 \\
\hline Chronic kidney disease & 21 & 4.71 \\
\hline Arrhythmia & 8 & 1.80 \\
\hline Stroke & 8 & 1.80 \\
\hline & Mean & SD \\
\hline Age (years) & 60.2 & 13.29 \\
\hline
\end{tabular}

$\mathrm{N}=$ absolute number; $\mathrm{SD}=$ standard deviation 


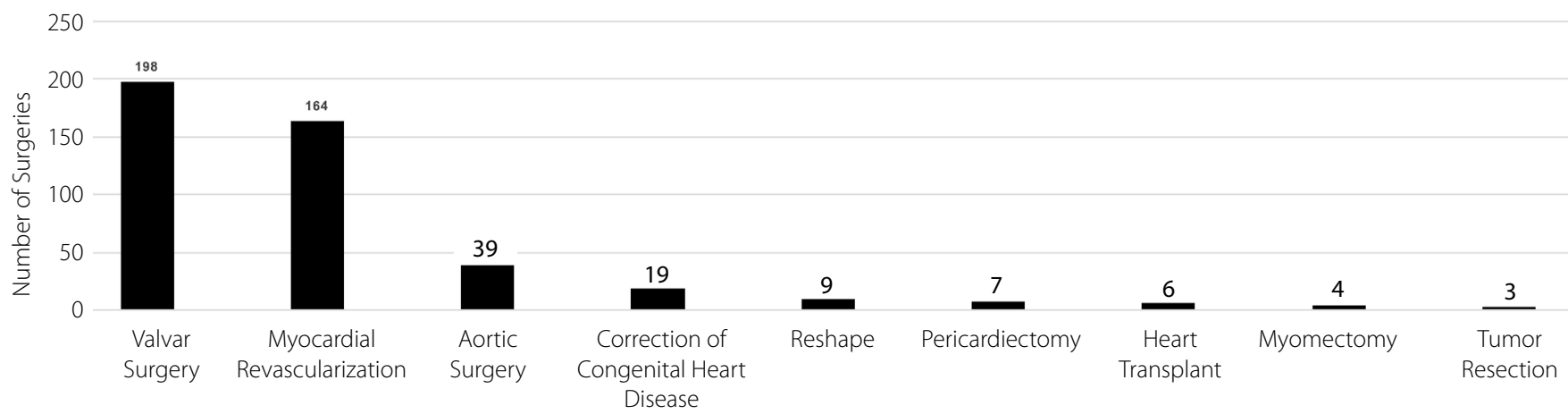

Fig. 1 - Types of surgeries performed.
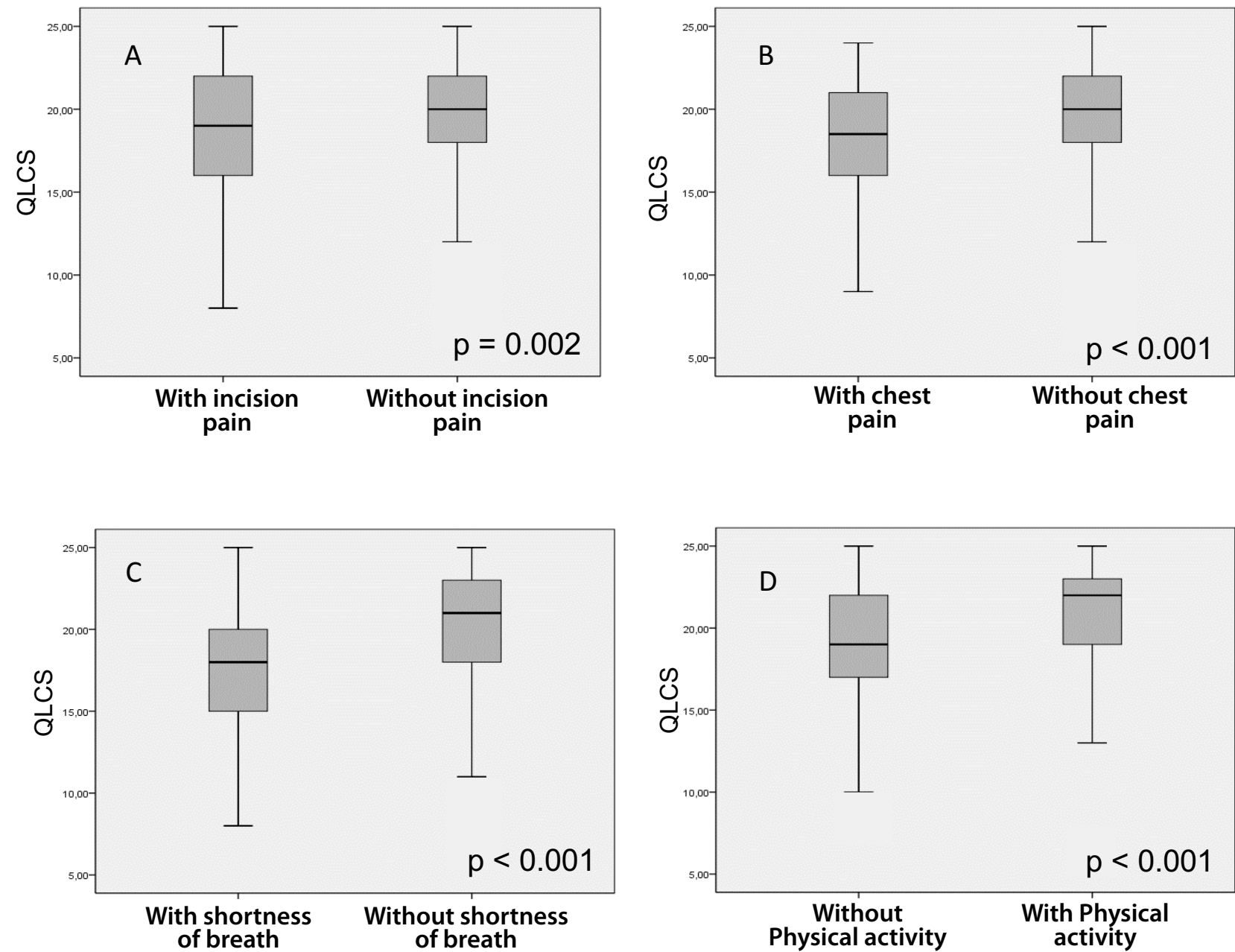

Fig. 2 - Comparison between the presence and absence of symptoms and the median obtained in the Quality of Life in Cardiovascular Surgery (QLCS) questionnaire showed in box-and-whisker plots. (A) Incision pain. (B) Chest pain. (C) Shortness of breath. (D) Physical activity. 
shortness of breath also affected the QLCS score, with a median of $18.0(\mathrm{LQ}=15.0 ; \mathrm{UQ}=20.25)$, compared to its absence, with a median of $21.0(\mathrm{LQ}=18.0 ; \mathrm{UQ}=23.0)$; $(P<0.001)$. So, in the evaluation of symptoms and QLCS scores, the presence of pain at incision $(P=0.002)$, chest pain $(P<0.001)$, and shortness of breath $(P<0.001)$ negatively affected QoL. On the other hand, other symptoms $(P=0.075)$, fainting $(P=0.50)$, palpitations $P=0.52)$, and infection $(P=0.48)$ weren't related to QoL. The return to physical activity was also significant, the group that performed physical activity had a higher median in the QLCS questionnaire (22.0; $\mathrm{LQ}=19.0 ; \mathrm{UQ}=23.0$ ) than the sedentary group (19.0; $L Q=17.0 ; \cup Q=22.0$ ), improving QoL $(P<0.001)$. When comparing the median of the group that returned to the routine $(20.0 ; \mathrm{LQ}=18.0 ; \mathrm{UQ}=22.0$ ) with the median of those who hadn't returned (17.0; $L Q=16.0 ; \cup Q=22.0$ ), it wasn't significant $(P=0.85)$. The comparison between the presence and absence of symptoms and the median obtained in the QLCS questionnaire is shown in box-and-whisker plots in Figure 2.

\section{DISCUSSION}

The present study represents an important advance to facilitate the assessment of QoL in postoperative cardiovascular surgery patients in Brazil and other countries. The QLCS is a new questionnaire with an internal consistency of 0.74 (Cronbach's alpha), being probably a good questionnaire to evaluate QoL in this population in a fast, easy, and objective way. Thus, it is possible to measure QoL in a practical way, even in countries where there aren't many resources and incentives for research, making possible a greater diffusion of QoL assessment by society, which contributes to the universality of knowledge. When Cronbach's is 0 , it indicates no internal consistency (i.e., none of the items are correlated with one another), whereas, when it is 1 , it reflects perfect internal consistency (i.e., all the items are perfectly correlated with one another). In practice, Cronbach's alpha of at least 0.70 has been suggested to indicate adequate internal consistency ${ }^{[14]}$. A low Cronbach's alpha value may be due to poor inter-relatedness between items; as such, items with low correlations with the questionnaire total score should be discarded or revised. On the other hand, an alpha value that is too high (more than 0.90) suggests that some questionnaire items may be redundant, and investigators may consider removing items that are essentially asking the same thing in multiple ways ${ }^{[12]}$.

The QoL in the postoperative period of cardiovascular surgery has been much studied in the international literature, which justifies even more the creation of this questionnaire. When referring to CABG, Aydin et al. ${ }^{[3]}$ compared pre and postoperative QoL in 120 patients over 65 years, and the SF-36 for postoperative results were higher than the SF-36 for preoperative results $(P<0.001)$. The same result of improvement in QoL with CABG was also demonstrated by Dal Boni et al. ${ }^{[4]}$ and Gois et al. ${ }^{[15]}$. In another study, Takiuti et al. ${ }^{[16]}$ compared clinical treatment, percutaneous coronary intervention, and CABG in 483 patients with coronary disease; there was an improvement in QoL in all domains and in the three therapeutic options, but the surgery offered better results after four years of follow-up.

In a Brazilian prospective cohort study with 44 elderly patients between 60 and 80 years of age, of both sexes, diagnosed with coronary artery disease, and undergoing CABG, from June 2010 to June 2011, a significant increase was observed in SF-36 scores between the pre and postoperative periods (three and six months) for the domains: functional capacity, pain, general health, vitality, and emotional aspect.

Albert et al. ${ }^{[17]}$ found that in the postoperative period of surgery for valvulopathy correction, the improvement of the gradient resulted in an improvement in the QoL. Another study, conducted by Ferreira et al[.18], showed that patients with cardiovascular valvar disease have a better QoL three to six months after surgery, compared to those awaiting surgical intervention.

Some studies addressed cardiovascular surgeries as a whole; a study published in 2008 by Colak et al. ${ }^{[199}$, which analyzed 111 patients in pre and postoperative periods and the health status of the patients one year after discharge, showed a statistically significant improvement, and the group of high-risk patients had the best benefit (EuroSCORE 6). Koch et al. ${ }^{[20]}$, in a study of 5581 patients, demonstrated that a lower socioeconomic status of the patient is associated with a lower QoL, while Noyez et al. ${ }^{[21]}$ concluded that QoL in cardiovascular surgery is overestimated, certainly for the elderly and those patients with preoperative low QoL.

Several authors, as described above, have already performed studies about the QoL assessment in the postoperative period of cardiovascular surgery, but a Brazilian study with a questionnaire created in Brazil, adapted to our reality, hasn't been conducted yet. In addition, this questionnaire was created to facilitate the acquisition of data, not only via personal contact, but also via telephone. By facilitating the way to obtain information about QoL, more people may be interested in using this parameter in the assessment of individuals' health, especially in the Brazilian context, where research isn't valued and resources are scarce.

The present study has some limitations. The first, it is a crosssectional analysis of a cohort. This was done only for the QLCS questionnaire's initial validation, the work with longitudinal analysis of the patients is already being elaborated and the testretest reliability will be made. The second, it is an unicentric study. On the other hand, once validated, we hope that many other scientific centers can take advantage of this questionnaire. The third refers to the limited number of patients, however, because it is a prospective study using the CEDREC registry, which began in June 2016, it was not possible to recruit a larger sample. Still, it was enough to perform the internal validation of the questionnaire, since only 63 patients were required. A fourth limitation is the possible bias associated with any questionnaire conducted via telephone. But it was created to be simpler and more objective to reduce this bias, and all phone calls were made by the same researcher. A fifth limitation could be the fact that we haven't chosen to create stricter exclusion criteria, excluding patients with lung diseases or neurodegenerative diseases. The QoL of these individuals would be invariably affected by these other conditions, and the insertion of these patients could provide a bias for the misunderstanding of adequately representing the impact of cardiovascular surgery on QoL. However, this could be done in a future research.

Finally, this is only the first validation step of this questionnaire, which presents adequate internal consistency. Subsequent steps 
will be performed to ensure the adequate use in the clinical practice of postoperative cardiovascular surgery patients.

\section{CONCLUSION}

The QLCS questionnaire is probably a good tool for the evaluation of QoL in the postoperative period of cardiovascular surgery, with adequate internal consistency, besides being an easy-to-apply instrument, which can even be done via telephone. The presence of chest pain, incision pain, and shortness of breath seems to be related to $\mathrm{Q} L$, making it worse, while return to physical activity seems to improve QoL.

\section{Authors' roles \& responsibilities}

MMKB Substantial contributions to the conception or design of the work; or the acquisition, analysis, or interpretation of data for the work; drafting the work or revising it critically for important intellectual content; final approval of the version to be published.

JLRO Substantial contributions to the conception or design of the work; or the acquisition, analysis, or interpretation of data for the work; drafting the work or revising it critically for important intellectual content; final approval of the version to be published.

LCBS Substantial contributions to the conception or design of the work; or the acquisition, analysis, or interpretation of data for the work; drafting the work or revising it critically for important intellectual content; final approval of the version to be published.

PSF Final approval of the version to be published.

VLA Final approval of the version to be published.

DJDT Final approval of the version to be published.

SKG Final approval of the version to be published.

MAS Substantial contributions to the conception or design of the work; or the acquisition, analysis, or interpretation of data for the work; drafting the work or revising it critically for important intellectual content; agreement to be accountable for all aspects of the work in ensuring that questions related to the accuracy or integrity of any part of the work are appropriately investigated and resolved; final approval of the version to be published.

\section{REFERENCES}

1. Faden R, Leplège A. Assessing quality of life. Moral implications for clinical practice. Med Care. 1992;30(5 Suppl):MS166-75.

2. GroupTW.The Development of the World Health Organization Quality of Life Assessment Instrument (the WHOQOL). In: Quality of life assessment: international perspectives. Berlin, Heidelberg: Springer; 1994. p.41-57.

3. Aydin S, Yavuz T, Duver H, Kutsal A. Quality of life in the elderly after coronary bypass surgery. Int Heart J. 2006;47(1):59-65.

4. Dal Boni ALM, Martinez JE, Saccomann ICRS. Quality of life of patients undergoing coronary artery bypass grafting. Acta Paul Enferm. 2013;26(6):575-80.

5. Ware JE Jr, Sherbourne CD. The MOS 36-item short-form health survey (SF-36). I. Conceptual framework and item selection. Med Care. 1992;30(6):473-83.

6. Ware J Jr, Kosinski M, Keller SD. A 12-item Short-Form Health Survey: construction of scales and preliminary tests of reliability and validity. Med Care. 1996;34(3):220-33.

7. Orley $\mathrm{JH}$, Kuyken W. Quality of life assessment: international perspectives. Proceedings of the joint meeting organized by the World Health Organization and the Foundation IPSEN in Paris, July 2-3 1993. Berlin: Springer-Verlag; 1994. 200p.

8. Development of the World Health Organization WHOQOL-BREF quality of life assessment. The WHOQOL Group. Psychol Med. 1998;28(3):551-8.

9. Green CP, Porter CB, Bresnahan DR, Spertus JA. Development and evaluation of the Kansas City Cardiomyopathy Questionnaire: a new health status measure for heart failure. J Am Coll Cardiol. 2000;35(5):1245-55.

10. Noyez L, Jager MJ, Markou AL. Quality of life after cardiac surgery: underresearched research. Interact Cardiovasc Thorac Surg. 2011;13(5):511-4.

11. Silva LDC, Linhares NS, Dias RS, Silva EL. Qualidade de vida de pacientes submetidos à cirurgia cardíaca: uma revisão sistemática. JMPHC J Manag Prim Health Care. 2012;3(2):96-101.

12. Tsang S, Royse CF, Terkawi AS. Guidelines for developing, translating, and validating a questionnaire in perioperative and pain medicine. Saudi J Anaesth. 2017;11(Suppl 1):S80-9.

13. Cronbach LJ. Coefficient alpha and the internal structure of tests. Psychom. 1951;16(3):297-334.

14. Nunnally JC. Psychometric theory. New York: McGraw Hill; 1967.

15. Gois CFL, Dantas RAS, Torrati FG. Qualidade de vida relacionada à saúde, antes e seis meses após a revascularização do miocárdio. Rev Gaúcha Enferm. 2009;30(4):700-7.

16. Takiuti ME, Hueb W, Hiscock SB, Nogueira CRSR, Girardi P, Fernandes F, et al. Qualidade de vida após revascularização cirúrgica do miocárdio, angioplastia ou tratamento clínico. Arq Bras Cardiol. 2007;88(5):537-44.

17. Albert A, Florath I, Rosendahl U, Hassanein W, Hodenberg EV, Bauer $\mathrm{S}$, et al. Effect of surgeon on transprosthetic gradients after aortic valve replacement with Freestyle stentless bioprosthesis and its consequences: a follow-up study in 587 patients. J Cardiothorac Surg. 2007;2:40

18. Ferreira AJS, Nunes AIM, Rodrigues EFP, Camarneiro APF. Qualidade de vida em doentes submetidos a cirurgia vascular cardíaca. Psicol Saúde Doenças. 2008;9(1):155-64.

19. ColakZ, Segotic I, Uzun S, Mazar M, Ivancan V, Majeric-Kogler V. Health related quality of life following cardiac surgery: correlation with EuroSCORE. Eur J Cardiothorac Surg. 2008;33(1):72-6.

20. Koch CG, Li L, Shishehbor M, Nissen S, SabikJ, Starr NJ, et al. Socioeconomic status and comorbidity as predictors of preoperative quality of life in cardiac surgery. J Thorac Cardiovasc Surg. 2008;136(3):665-72.

21. Noyez L. Is quality of life post cardiac surgery overestimated? Health Qual Life Outcomes. 2014;12:62. 